\title{
Scientists
}

\section{Climbing the Hill}

\author{
John T. Lis \\ Barbara McClintock Professor of Molecular Biology and Genetics, Cornell University, Ithaca, New York
}

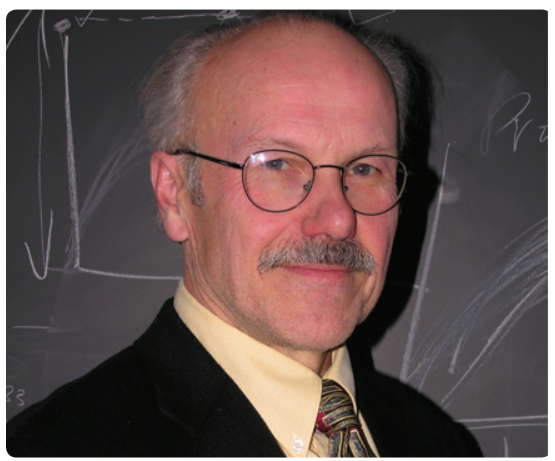

John Lis's research into the mechanisms of transcriptional regulation and his development of new techniques caught our attention. Curious to know more, BioTechniques contacted him to find out about the ambition, character, and motivation that led to his success.

\section{What has been your most significant scientific contribution so far?}

I arrived at Cornell University shortly after finishing a postdoctoral fellowship in Dave Hogness's lab at Stanford. At the time, the Hogness lab was using early gene isolation methods and doing recombinant DNA work, and I was eager to apply these methods to eukaryotic systems. At Cornell, I gathered my students together to talk about my goals; during that conversation, a very bright student, Dave Gilmour, asked why everyone was looking at DNA and genes, but ignoring the proteins that interacted with them. I thought about his question quite seriously and decided that we should look into it. However, at that time, there wasn't a method available for detecting protein-DNA interactions in vivo, so we set out to develop a technique.

In the beginning, we tried fixing proteins onto DNA and then pulling out the DNA sequences, but this turned out to be impractical. We worked for three years without a publishable result. Still, some of our best times were spent on Saturday mornings discussing all the failed experiments of the week and planning what to do next. In a bizarre way, it was fun.

After working on developing this method for some time, we decided to try a different approach. We used antibodies to pull out the proteins and then used sensitive hybridization methods for looking at particular DNA sequences. In the end, what we came up with was the first strategy for studying specific protein-DNA interactions in vivo. Our method, which was based on UV light crosslinking of proteins to DNA, was a precursor to chromatin immunoprecipitation (ChIP). UV light is a zero length crosslinker, so it requires contact between the proteins and nucleic acids. That method wasn't as versatile as ChIP, which uses formaldehyde to crosslink everything, but it was more specific for direct protein-DNA interactions.

In the course of your research, have you encountered anything that really surprised you?

While developing the crosslinking method, we studied the interactions between polymerase and heat shock genes. At the time, everyone thought gene regulation required recruitment of the transcription machinery to the gene, so it was a surprise when we found RNA polymerase II was already on the promoter before the gene was induced.

We did a lot of follow-up experiments and studies using other methods to finally convince ourselves that our interpretation was correct and this polymerase was paused on the promoter. It turns out that polymerase pausing on promoter regions is a general mechanism and rate limiting step of gene regulation in metazoans. Since that time, polymerases have been shown to be piled up on promoters of many genes; this is a common method of gene regulation in eukaryotes.

Are you working on any "pet projects" outside of your main research focus?

Over the past 14 years, we have been developing new reagents to inhibit macromolecular interactions. We focus on RNA aptamers, which are RNAs selected to bind a specific target, and because they are nucleic acids, they can be amplified. So we make large libraries of RNAs in vitro, select and amplify those that bind specific proteins, and then use these RNAs to detect the corresponding proteins or inhibit their interactions.
What is the potential for the RNA aptamers you are developing?

My dream is that by using reagents like RNA aptamers - molecules capable of high affinity, high specificity interactions with particular targets - we can routinely develop detectors and inhibitors for specific biochemical macromolecular components that will be extremely valuable for dissecting the mechanisms by which those macromolecules work. And since they can serve as inhibitors, there is also the potential for drug development. For example, an aptamer we isolated against the Heat Shock Factor also has the ability to inhibit cancer cell growth.

Although aptamers were developed as a side project, they serve our primary focus, which is developing approaches to uncover biological mechanisms in living cells. We develop and use high resolution assays of specific biochemical processes in vivo, perturb specific macromolecular interactions, and then measure the biochemical events again to see what has changed. Doing this in a systematic way allows us to clarify the mechanisms involved in the system being studied. Although we focus on transcriptional regulation, we hope that the tools and strategies developed in the lab will be useful for studies in other fields of biology as well.

\section{What motivates you to develop new methods?}

I find that new methods provide new views of biological processes and potentially new solutions to key questions. So I like to look for limitations in our technological approaches and then figure out a way to climb the hill to see thrilling new vistas beyond those limitations.

Interviewed by Kristie Nybo, Ph.D. Image courtesy of Digbijar (Jay) Mahat. 㳡

BioTechniques 52:15 (January 2012)

doi 10.2144/000113795

To purchase reprints of this article,

contact:biotechniques@fosterprinting.com 